\title{
Concomitant boost IMRT-based neoadjuvant chemoradiotherapy for clinical stage II/III rectal adenocarcinoma: results of a phase II study
}

Ji Zhu ${ }^{1,5 \dagger}$, Fangqi Liu ${ }^{2,5 \dagger}$, Weilie Gu ${ }^{2,5}$, Peng Lian ${ }^{2,5}$, Weiqi Sheng ${ }^{3,5}$, Junyan Xu ${ }^{4,5}$, Gang Cai ${ }^{1,5}$, Debing Shi ${ }^{2,5}$, Sanjun Cai ${ }^{2,5^{*}}$ and Zhen Zhang ${ }^{1,5^{*}}$

\begin{abstract}
Aim: This study was designed to evaluate the efficacy and toxicities of concomitant boost intensity-modulated radiation therapy (IMRT) along with capecitabine and oxaliplatin, followed by a cycle of Xelox, in neoadjuvant course for locally advanced rectal cancer.

Materials and methods: Patients with histologically confirmed, newly diagnosed, locally advanced rectal adenocarcinoma (cT3-T4 and/or cN+) located within $12 \mathrm{~cm}$ of the anal verge were included in this study. Patients received IMRT to the pelvis of $50 \mathrm{~Gy}$ and a concomitant boost of $5 \mathrm{~Gy}$ to the primary tumor in 25 fractions, and concurrent with oxaliplatin ( $50 \mathrm{mg} / \mathrm{m}^{2} \mathrm{~d} 1$ weekly) and capecitabine $\left(625 \mathrm{mg} / \mathrm{m}^{2}\right.$ bid d1 -5 weekly). One cycle of Xelox (oxaliplatin $130 \mathrm{mg} / \mathrm{m}^{2}$ on $\mathrm{d} 1$ and capecitabine $1000 \mathrm{mg} / \mathrm{m}^{2}$ twice daily $\mathrm{d} 1-14$ ) was given two weeks after the completion of chemoradiation, and radical surgery was scheduled eight weeks after chemoradiation. Tumor response was evaluated by tumor regression grade (TRG) system and acute toxicities were evaluated by NCl-CTC 3.0 criteria. Survival curves were estimated using the Kaplan-Meier method and compared with Log-rank test.

Results: A total of 78 patients were included between March 2009 and May 2011 (median age 54 years; 62 male). Seventy-six patients underwent surgical resection. Twenty-eight patients underwent sphincter-sparing lower anterior resection and 18 patients (23.7\%) were evaluated as pathological complete response ( $p C R)$. The incidences of grade 3 hematologic toxicity, diarrhea, and radiation dermatitis were 3.8\%, 10.3\%, and 17.9\%, respectively. The three-year LR (local recurrence), DFS (disease-free survival) and OS (overall survival) rates were $14.6 \%, 63.8 \%$ and $77.4 \%$, respectively. Initial clinical T stage and tumor regression were independent prognostic factors to DFS.
\end{abstract}

Conclusion: An intensified regimen of concomitant boost radiotherapy plus concurrent capecitabine and oxaliplatin, followed by one cycle of Xelox, can be safely administered in patients with locally advanced rectal cancer, and produces a high rate of pCR. A prognostic score model is helpful to distinguish different long-term prognosis groups in early stage.

Keywords: Rectal cancer, Intensity-modulated radiation therapy, Concomitant boost, Neoadjuvant chemoradiotherapy

\footnotetext{
* Correspondence: caisanjun@csco.org.cn; zhenzhang6@gmail.com

${ }^{\dagger}$ Equal contributors

${ }^{2}$ Department of Colorectal Surgery, Fudan University Shanghai Cancer Center, No. 270, Dong'An Road, Shanghai 200032, China

'Department of Radiation Oncology, Fudan University Shanghai Cancer

Center, No. 270, Dong'An Road, Shanghai 200032, China

Full list of author information is available at the end of the article
} 


\section{Introduction}

Preoperative chemoradiotherapy (CRT) followed by total mesorectal excision (TME) is the standard treatment for patients with locally advanced rectal cancer (LARC). Some significant benefits of neoadjuvant CRT, such as better local control and sphincter preservation, have been shown in patients with stage II/III rectal cancer [1-3].

To obtain a better tumor response, elevating treatment dose has been considered a feasible method. EORTC22921 and FFCD9203 studies showed that preoperative radiotherapy combined with fluorouracil (5-FU) can significantly improve the treatment effect compared with radiotherapy alone $[3,4]$. In a retrospective analysis of 3,157 patients enrolled in seven randomized Phase III trials and 45 Phase II trials, the use of continuous infusion 5-FU, a second drug based on 5-FU and a higher radiation dose was associated with higher rates of $\mathrm{pCR}$ [5]. However, the next five randomized phase III trials, ACCORD 12/0405-Prodige 2 [6], STAR-01 [7], NSABP R-04 [8], CAO/ARO/AIO-04 [9] and PETACC-6 [10], demonstrated conflicting results as to whether oxaliplation increased the rate of $\mathrm{pCR}$.

In our center, most patients receiving neoadjuvant CRT were clinical $\mathrm{T} 4$ or $\mathrm{N}+$, and might have more opportunities to benefit from a high intensity treatment, whether chemotherapy or radiotherapy [11-13]. To decrease the additional toxicities from a high-dose treatment, especially diarrhea, intensity modulated radiotherapy (IMRT) was used to lessen radiation-associated toxicities by decreasing the volume of high irradiation dose of surrounding normal tissues [14-16]. IMRT allows higher radiation doses to be focused on regions within the tumor while minimizing the dose to surrounding normal critical structures. The data from dosimetric studies of IMRT in rectal cancer are encouraging. Compared to conventional 2D or 3D radiation therapy, IMRT showed similar target coverage with reduced dose to the small bowel, bladder, pelvic bone and femoral heads $[14,17,18]$. By decreasing the dose delivered to normal structures, IMRT may provide a potential for increasing treatment dose to improve tumor response.

Therefore, we designed this study to examine the use of IMRT, escalating the primary lesion's dose to $55 \mathrm{~Gy}$ together with the whole pelvis dose of 50 Gy in 25 fractions, along with weekly capecitabine and oxaliplatin. Two weeks after the end of chemoradiotherapy, a cycle of Xelox (capecitabine and oxaliplatin) was prescribed before surgery. The efficacy and toxicity of this modality were evaluated to explore the feasibility of high-dose intensity in preoperative treatment. This phase II study was approved by our institutional review board.

\section{Materials and methods Eligibility criteria}

Patients with histologically confirmed, newly diagnosed, locally advanced rectal adenocarcinoma (cT3-T4 and/or
$\mathrm{cN}+$ ) located within $12 \mathrm{~cm}$ from the anal verge were included in this study at the Fudan University Shanghai Cancer Center. All patients were $\geq 18$ years of age and had a Karnofsky Performance Status score of $\geq 60$, no evidence of distant metastases, adequate bone marrow function (leukocyte count $>4,000 / \mathrm{mL}$ and platelet count $>100,000 / \mathrm{mL}$ ), and adequate renal and hepatic function (creatinine clearance $>50 \mathrm{~mL} / \mathrm{min}$ and bilirubin $\leq 2 \mathrm{mg} / \mathrm{mL}$ ). Patients were excluded if they were older than 75 years of age, had undergone previous pelvic radiotherapy or previous chemotherapy, or had previous or synchronous tumors other than nonmelanoma skin cancer. Patients suffering of the following medical conditions were also ineligible: ischemic heart disease, inflammatory bowel disease, malabsorption syndrome, peripheral neuropathy, or psychological disorders. Signed informed consent was obtained from all patients before inclusion on this study. The institutional review board of Fudan University approved the study.

\section{Baseline evaluation}

Pretreatment evaluation was performed within two weeks before initiation of chemoradiation. The evaluation included a complete history and physical examination, including digital rectal examination, complete blood count, hepatic and renal function tests, tumor marker measurement, colonoscopy and biopsy, computed tomography (CT) of the thorax and abdomen, magnetic resonance imaging (MRI) of the pelvis, and, in selected patients, endorectal ultrasound. All patients were clinically staged with the AJCC 7th version manual.

\section{Combined chemoradiotherapy}

\section{Intensity modulated radiation therapy (IMRT)}

All patients were immobilized in the prone position using a belly board and underwent a non-contrast-enhanced, planning CT with 5-mm slices from the L3-L4 junction to $2 \mathrm{~cm}$ below the perineum. The image data were transferred to the PINNACLE planning system (Philips Radiation Oncology Systems, Milpitas, CA). The definitions of volumes were in accordance with the ICRU Report \#83 [19]. The gross tumor volume (GTV) was defined as all known gross disease determined from $\mathrm{CT}$ and MRI. The clinical target volume 1 (CTV1) included the gross tumor volume and the corresponding mesorectum plus $2 \mathrm{~cm}$ cranio-caudally. The CTV2 included the CTV1 plus the entire mesorectum, entire pre-sacral space, internal iliac nodes and high-risk anatomical and nodal sub-sites, based on the distance of the tumor from the anal margin [20]. Based on our institution set-up data, the planning target volume (PTV) was defined as the CTV with $10-\mathrm{mm}$ margins superiorly and inferiorly and 8-mm margins in all other directions. Organs at risk (OARs) were contoured as follows: 1) the small intestine was defined as all intestinal 
loops below the sacral promontory (rectosigmoid junction excluded); 2) femoral heads were contoured from the cranial extremity to the level of the lower margin of ischial tuberosities; and 3) the bladder was contoured entirely with no distinction between the wall and its content [16]. The IMRT plans were generated using the inverse planning module of PINNACLE for a 6-MV liner accelerator, with five to seven coplanar fields.

The planned doses to the PTV1 and PTV2 were 55 Gy and $50 \mathrm{~Gy}$, respectively, in 25 fractions, five times per week (Monday through Friday) for five weeks. The D2\%, D50\%, and D98\% to PTV1 and PTV2 were set at 52.25 Gy and 55 Gy, 57.75 Gy and 47.5 Gy, and 50 Gy and $52.5 \mathrm{~Gy}$, respectively. The doses of the OARs were set as low as possible and had to at least meet the following constraints: bladder, $\geq 45$ Gy in $15 \%$ volume and $\geq 40$ Gy in $40 \%$ volume; femoral heads, $\geq 45$ Gy in $25 \%$ volume and $\geq 40$ Gy in $40 \%$ volume; and small bowel, $\geq 45$ Gy in 65 cc volume, $\geq 40$ Gy in 100 cc volume, and $\geq 35$ Gy in 180 cc volume.

The positioning and isocenter of each patient were verified on electronic portal imaging device (EPID) films for the anterior and lateral gantry positions by visually comparing the digitally reconstructed radiographs.

\section{Concurrent and neoadjuvant chemotherapy}

Capecitabine combined with oxaliplatin was administered concurrently with pelvic radiation. Capecitabine was given at a dose of $625 \mathrm{mg} / \mathrm{m}^{2}$ twice daily from Monday to Friday throughout the entire course of IMRT. Oxaliplatin at a dose of $50 \mathrm{mg} / \mathrm{m}^{2}$ was administered weekly during the five-week course of radiotherapy. Two weeks after concurrent chemoradiation, one cycle of Xelox (oxaliplatin $130 \mathrm{mg} / \mathrm{m}^{2}$ on $\mathrm{d} 1$ and capecitabine $1000 \mathrm{mg} / \mathrm{m}^{2}$ twice daily d1-14) was administered (Figure 1).

\section{Surgery and histopathology}

Surgery was scheduled eight weeks after the completion of CRT. Total mesorectal excision (TME) was mandatory, whereas the form of surgery (anterior resection or abdominal-perineal resection) and whether a temporary colostomy should be performed were decided by the surgeon. All lymph nodes were examined according to standard procedures. If the number of lymph nodes was less than 12, two pathologists were needed to sign to ensure the reliability of the detection result. The circumferential rectal margin (CRM) was assessed according to the method of Quirke et al. [2], and a margin of $<1 \mathrm{~mm}$ was considered CRM-positive. All sections of the surgical specimens were reviewed by two pathologists. The pathologic stage (ypTN) was recorded according to the Union for International Cancer Control (UICC) TNM system. Tumor regression grading (TRG) was evaluated according to the criteria by Dworak et al. as follows [21]: Grade 0, no regression; Grade 1, dominant tumor mass with obvious fibrosis and/or vasculopathy; Grade 2, dominant fibrotic changes with few tumor cells or groups (easy to find); Grade 3, very few (difficult to find microscopically) tumor cells in fibrotic tissue with or without mucous substance; and Grade 4, no tumor cells, only a fibrotic mass (total regression or response).

\section{Adjuvant chemotherapy and follow-up}

All patients were recommended to receive postoperative chemotherapy regardless of pathological stages. Adjuvant chemotherapy was recommended consisting of five cycles of Xelox. Patient follow-up was scheduled every three months during the first two years, and then every six months over the next three years. After five years, the frequency of follow-up was extended to once each year.

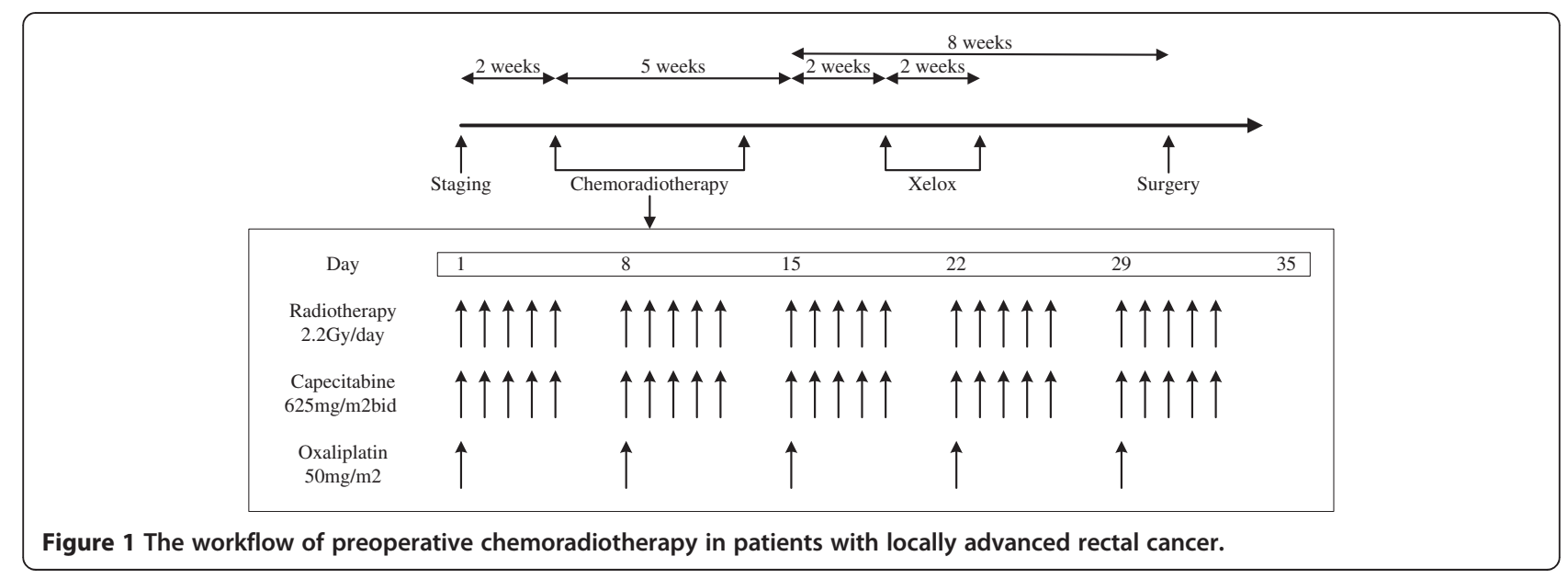




\section{Toxicity and measurement}

Toxicities were evaluated and recorded weekly according to the CTC 3.0 criteria. If grade 3 toxicities occurred, the physicians determined causes and decided the response. In general, the sequence of dose reduction or suspension moved from oxaliplatin to capecitabine to radiotherapy, unless an adverse effect was strongly associated with a particular treatment.

\section{Endpoints and statistics}

The primary endpoint for this trial was $\mathrm{pCR}$ rate. This study was a phase II trial of 78 patients to evaluate the treatment feasibility and efficacy of this dosing regimen. Based on a literature review, the pCR rate is approximately $10-15 \%$ for patients treated with neoadjuvant CRT. We determined that an experimental arm with a pCR rate of at least $18 \%$ would merit further study. In this study, if more than 17 cases were evaluated as pCR, we had $85 \%$ power to reject the null hypothesis that our strategy could not reach the pCR of $18 \%$, with a type I error level of 5\%. Secondary endpoints included safety, sphincter preservation rate, TRG, LR (local recurrence), DFS (disease-free survival) and OS (overall survival). Sphincter preservation was defined as any procedure in which the rectal tumor was removed while leaving behind the anal sphincter.

All characteristics were described by the frequency for classified variables, by mean and standard deviations for normal distributional continuous data, and by the median for non-normal distributional continuous data.

Survival time was calculated from the beginning of CRT to the date of event or the last follow-up. Survival curves were estimated using the Kaplan-Meier method and compared with Log-rank test. Cox proportional hazards regression was used for multivariate modeling and for examining the prognostic significance of the variables identified in the models. $\mathrm{P}$ values of less than 0.05 were taken to indicate statistically significant differences.

\section{Results}

\section{Clinical characteristics}

Between March 2009 and May 2011, a total of 78 patients were included in the study. All patients were diagnosed with locally advanced rectal cancer: 50 with cT3 and 28 with cT4 primary tumor. Lymph node involvements were detected in 75 patients. Of the total 78 patients, 62 were men and 16 were women; the median age was 54 years (range, $30-76$ years). Fifty-six patients $(71.8 \%$ ) had tumors located $\leq 5 \mathrm{~cm}$ from the anal verge (Table 1 ).

\section{Treatment compliance and acute toxicities}

All patients completed the prescribed radiation treatment to a total dose of $55 \mathrm{~Gy}$ in 25 fractions. The median total radiation duration was 37 days (range, 33-41). All patients
Table 1 Demographic and clinical features for all patients

\begin{tabular}{lcc}
\hline & $\mathbf{n}$ & $\%$ \\
\hline Gender & & \\
Male & 62 & 79.5 \\
Female & 16 & 20.5 \\
Age, years & & \\
$\quad$ Median (min-max) & & \\
Distance from anal verge & & \\
S5 cm & 56 & 71.8 \\
$>5$ cm & 22 & 28.2 \\
CT stage & & \\
T3 & 50 & 64.1 \\
T4 & 28 & 35.9 \\
CN stage & & \\
N0 & 3 & 3.8 \\
N1 & 34 & 43.6 \\
N2 & 41 & 52.6 \\
Total & 78 & 100.0 \\
\hline
\end{tabular}

completed five weeks of capecitabine, and 48 cases received five cycles oxaliplatin and the rest received four cycles. In addition, all patients received a scheduled single cycle of Xelox two weeks after the completion of chemoradiotherapy without dose adjustment.

Most of the adverse events during CRT were mild (grade 1 or 2). No grade 4-5 toxicities were observed. The most common grade 3 toxicity was radiation dermatitis (17.9\%), while grade 3 diarrhea and hematological toxicities were evaluated in eight $(10.3 \%)$ and three cases $(3.8 \%)$ (Table 2).

\section{Surgical procedures and pathological response}

Seventy-six patients underwent a surgical resection according to the schedule. One patient refused surgery because of good response, and another case did not receive an operation because of being evaluated as unresectable lesions. The median interval between the completion of CRT and primary tumor surgery was 52 days (range, 46-67 days). Twenty-eight patients (36.8\%) underwent sphincter-sparing

Table 2 Toxicity during the course of chemoradiation

\begin{tabular}{|c|c|c|c|c|c|c|}
\hline & \multicolumn{2}{|c|}{ Grade 1} & \multicolumn{2}{|c|}{ Grade 2} & \multicolumn{2}{|c|}{ Grade 3} \\
\hline & $\mathrm{n}$ & $\%$ & $\mathrm{n}$ & $\%$ & $n$ & $\%$ \\
\hline Diarrhea & 15 & $19.2 \%$ & 11 & $14.1 \%$ & 8 & $10.3 \%$ \\
\hline Hematologic & 13 & $16.7 \%$ & 3 & $3.8 \%$ & 3 & $3.8 \%$ \\
\hline Fatigue & 10 & $12.8 \%$ & 5 & $6.4 \%$ & 3 & $3.8 \%$ \\
\hline Radiation dermatitis & 20 & $25.6 \%$ & 16 & $20.5 \%$ & 14 & $17.9 \%$ \\
\hline Neurosensory & 3 & $3.8 \%$ & 1 & $1.3 \%$ & 0 & $0.0 \%$ \\
\hline Hand-foot syndrome & 0 & $0.0 \%$ & 1 & $1.3 \%$ & 0 & $0.0 \%$ \\
\hline
\end{tabular}


lower anterior resection. All pathological features are listed in Table 3. ypT0 and ypN0 were found in 18 (23.7\%) and $47(61.8 \%)$ patients, respectively, with 18 patients (23.7\%) showing pCR. TRG information was available in pathologic examination for all 76 patients receiving surgery. The TRG stage was Grade 4 (pCR) in 18 patients, Grade 3 in 34 patients, Grade 2 in 17 patients, and Grade 1 in 7 patients. All cases were divided into two subgroups: good responders (defined as TRG 3-4) or poor responders (defined as TRG $1-2)$. More than or equal to 12 lymph nodes were found in half of the patients. Lymphatic/vascular invasion and neural invasion were confirmed in five and eight cases, respectively (Table 3). The overall rate of postoperative complications

Table 3 Surgical procedure and pathological findings

\begin{tabular}{|c|c|c|}
\hline & $\mathrm{n}$ & $\%$ \\
\hline \multicolumn{3}{|l|}{ Surgery } \\
\hline Anterior resection & 28 & $36.8 \%$ \\
\hline Abdominal perineal resection & 46 & $60.5 \%$ \\
\hline Hartmann & 2 & $2.6 \%$ \\
\hline \multicolumn{3}{|l|}{ Lymphatic or vascular invasion } \\
\hline No & 71 & $93.4 \%$ \\
\hline Yes & 5 & $6.6 \%$ \\
\hline \multicolumn{3}{|l|}{ Neural invasion } \\
\hline No & 68 & $89.5 \%$ \\
\hline Yes & 8 & $10.5 \%$ \\
\hline \multicolumn{3}{|l|}{ CRM } \\
\hline Negative & 76 & $100.0 \%$ \\
\hline Positive & 0 & $0.0 \%$ \\
\hline \multicolumn{3}{|l|}{ ypT stage } \\
\hline TO & 18 & $23.7 \%$ \\
\hline $\mathrm{T} 1$ & 10 & $13.2 \%$ \\
\hline $\mathrm{T} 2$ & 20 & $26.3 \%$ \\
\hline $\mathrm{T} 3$ & 26 & $34.2 \%$ \\
\hline T4 & 2 & $2.6 \%$ \\
\hline \multicolumn{3}{|l|}{ ypN stage } \\
\hline No & 47 & $61.8 \%$ \\
\hline N1 & 19 & $25.0 \%$ \\
\hline N2 & 10 & $13.2 \%$ \\
\hline \multicolumn{3}{|l|}{ Examined lymph nodes } \\
\hline Median (Min-max) & & $12(2-35)$ \\
\hline \multicolumn{3}{|l|}{ TRG Score } \\
\hline 4 & 18 & $23.1 \%$ \\
\hline 3 & 34 & $43.6 \%$ \\
\hline 2 & 17 & $21.8 \%$ \\
\hline 1 & 7 & $9.0 \%$ \\
\hline Total & 76 & $100.0 \%$ \\
\hline
\end{tabular}

CRM: circumferential resection margin. TRG: tumor regression grade. was $17.1 \%$. Delayed sacral-wound healing, postoperative bleeding and anastomotic leakage occurred in 9, 3 and 1 patients, respectively.

\section{Follow-up}

With a median follow-up of 30 months (range, 948 months), 10 patients were diagnosed with local recurrence and 19 patients were confirmed with distant metastases (5 in the liver, 13 in the lung, and 1 in bone). Fourteen patients died of rectal cancer. For the two patients that did not receive surgery, one patient was confirmed of tumor failure at 27 months and died 28 months after the beginning of CRT, and the other patient did not present any evidence of failure at the last visit of 9 months. The 3-year LR, DFS and OS rates were $14.6 \%, 63.8 \%$ and $77.4 \%$, respectively (Figure 2).

\section{Univariate and multivariate analysis for $L R$ and DFS}

All potential prognostic factors, including age, gender, distance from anal verge, $\mathrm{cT}$ stage, $\mathrm{cN}$ stage, ypT stage, ypN stage and TRG score were evaluated using the Kaplan-Meier method (compared with Log-rank test). cT stage and pCR status demonstrated a correlation with LR (Figure 3). No LR was observed in pCR cases. In the next multivariate Cox regression analysis, only cT stage was left in the model.

cT stage, ypT stage and TRG score exhibit a correlation with DFS. YpT stage was excluded in the further multivariate Cox regression analysis. Hazard ratios of these two factors (cT stage and TRG score) were very close to one another (Table 4). A prognostic scoring system was produced, with each of the two unfavorable prognostic factors allocated one point, including cT4 stage and poor responder. Use of the scoring system led

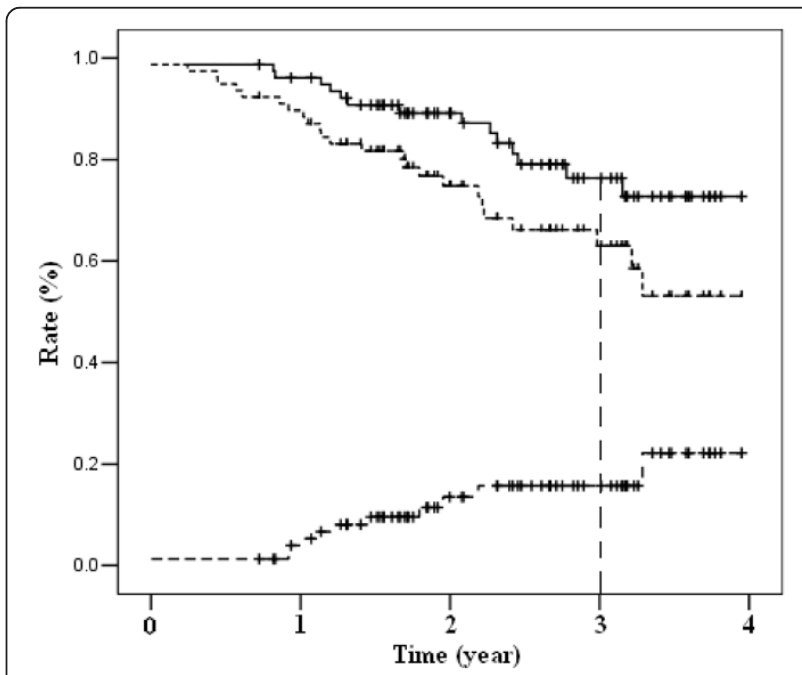

Figure 2 Survival curves of local recurrence, overall survival (OS) and disease-free survival (DFS). 


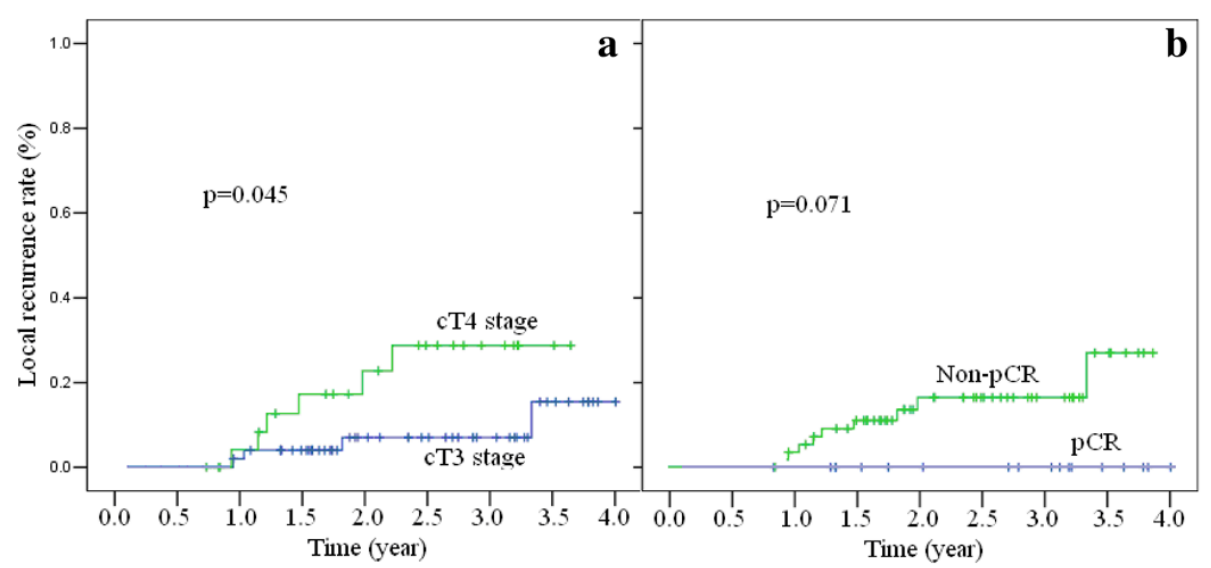

Figure 3 Local recurrence rates by clinical T stage (panels a) and pCR or not (panels b). pCR: pathological complete response.

to the identification of three risk groups: low risk (score of 0 ), intermediate risk (score of 1), and high risk (score of 2). The three-year DFS rates were significantly different among the groups: the low-risk group, $81.1 \%$; intermediate-risk group, $60.0 \%$; and the high-risk group, $28.6 \%$ $(P=0.000$, Figure 4$)$.

\section{Discussion}

Our study findings demonstrated that the IMRT technique, which decreases radiation-induced toxicities by a lower high-dose irradiation volume, and an intensified preoperative CRT followed by a cycle of Xelox resulted in a pCR rate of $24.7 \%$. These results are higher than those of studies on preoperative conventional CRT concurrent with oxaliplatin (15-20\%) [6-10]. Regarding toxicities, however, the high rate of $\mathrm{pCR}$ did not translate into high local control compared to other trials. This may be attributed to two factors, First, our study had a small sample size, and therefore some certain remains. Second, the percentage of cT4 and $\mathrm{cN}+$ tumors in our study was significantly higher than some other trials (cT4: $35.9 \%$ vs. $5-15 \%$, cN+: $96.2 \%$ vs. $40-70 \%$, respectively) [1,3,5,7-11]. Our study found that the incidence of grade 3 diarrhea, hematologic toxicity, and radiation dermatitis was $10.3 \%, 3.8 \%$, and $17.9 \%$, respectively. Consistent with our previous study [22], the incidences of diarrhea and hematologic toxicity were slightly lower

Table 4 The values of $\beta$, hazard ratio and $P$ values in Cox multivariate regression model disease-free survival

\begin{tabular}{llccc}
\hline & & $\boldsymbol{\beta}$ & Hazard ratio & $\boldsymbol{P}$ value \\
\hline LR & Clinical T stage (3 vs. 4) & 1.23 & 3.43 & 0.05 \\
DFS & TRG score (3/4 vs. 1/2) & 1.36 & 3.90 & 0.00 \\
& Clinical T stage (3 vs. 4) & 1.11 & 3.04 & 0.01 \\
\hline
\end{tabular}

LR: local recurrence.

DFS: disease-free survival.

TRG: tumor regression grade. compared with other reported stage III clinical trials. A significant increase in the incidence of radiation dermatitis was observed in our study, which might be attributed to a lower irradiation field due to a distal rectal tumor location in most cases. Finally, with a median follow-up of 30 months, our data showed that the baseline $\mathrm{T}$ stage and treatment response were associated with long-term prognosis. Patients with cT4 stage had a higher chance of LR and distance metastases, but no LR was observed in $\mathrm{pCR}$ cases, regardless of initial T stage. In addition, baseline $\mathrm{T}$ stage and tumor response had equal effects to predict DFS. Patients with cT3 and good response had a three-year DFS of $81.1 \%$, but for those with cT4 and poor response, the three-year DFS declined to $28.6 \%$.

The IMRT technique has been used widely in some solid tumors. In our study, IMRT was used to concomitantly boost the total irradiation dose to 55 Gy for the gross tumor. The rationale was based on several dosimetric studies that showed that IMRT could significantly decrease the surrounding organs' high-dose irradiation volume, especially the small bowel, compared with conventional radiotherapy or 3DCRT $[14,15]$. Therefore, IMRT provided the potential of elevating the dose to improve the tumor response. This is consistent with results from Hartley's meta-analysis, which included a total of 3157 rectal cancer patients [5]. Our previous study of stage IV rectal cancer demonstrated the feasibility to deliver 45 Gy to the pelvis and a concomitant 10 Gy boost to the gross tumor using IMRT [23].

Oxaliplatin is an effective drug for colorectal cancer when combined with 5-FU. However, the results of five recent large sample phase III trials are unclear as to whether oxaliplatin is an appropriate radiation sensitizer. Three studies, including NSABP R-04 [8], STAR-01 [7] and PETACC-6 [10], all reported that additional oxaliplation based on conventional CRT failed to increase pCR rate and caused more toxicities, especially diarrhea. 


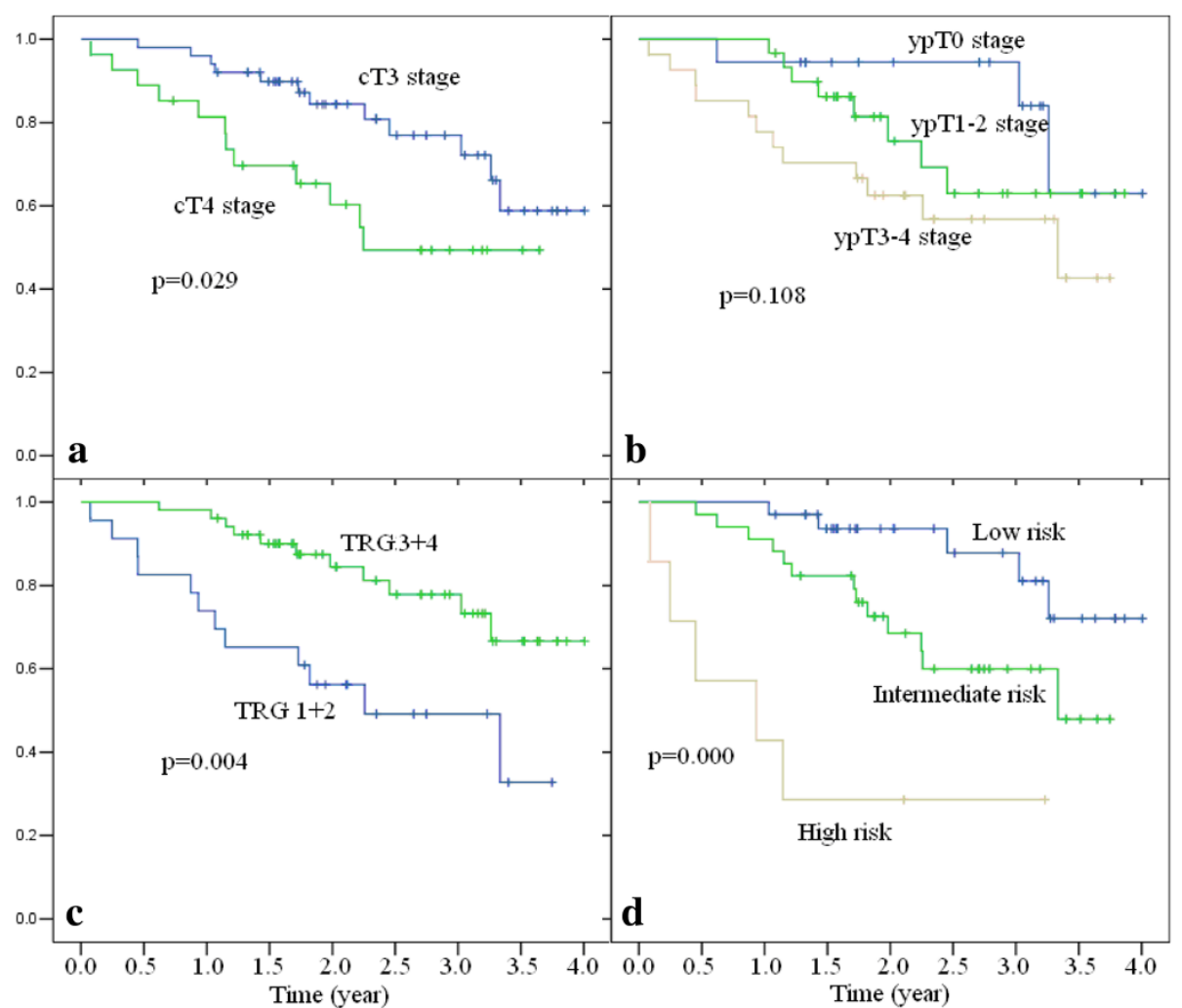

Figure 4 Disease-free survival rates by clinical T stage (panels a), ypT stage (panels b), tumor regression (panels c) and prognostic score (panels d). TRG: tumor regression grade.

The ACCORD 12/0405-Prodige 2 trial also reported a higher toxicity rate in the oxaliplatin group, together with a significantly higher $\mathrm{pCR}$ rate [6]. The $\mathrm{CAO/ARO/}$ AIO-04 trial was the only phase III trial supporting additional oxaliplatin in preoperative CRT, which showed a better tumor regression in the oxaliplatin group without any additional toxicities [9]. However, our study demonstrated an encouraging $\mathrm{pCR}$ and tumor shrinkage, without high incidence of toxicities. This also illustrated that IMRT was an effective method to offset toxicities induced by high-dose CRT.

In several previous studies, early surrogate indicator was focused to help to decide the strategy of adjuvant therapy. Tumor regression after CRT, especially pCR, was regarded as an important prognostic factor. The EORTC 22921 trial showed that patients with good response had a significantly good prognosis compared with those with poor response [24]. Capirci's retrospective study of 566 patients with $\mathrm{pCR}$ also demonstrated an encouraging prognosis [25]. In a retrospective study by MD Anderson Cancer Center, tumor response was associated with the five-year recurrence free survival, distant-metastasis rate and LR [26]. Thus, after neoadjuvant therapy, conventional adjuvant chemotherapy (oxaliplatin plus fluorouracil) might be over-treatment to patients with good prognosis, but no use to poor responders. Based on our data, a prognostic score model including initial clinical $\mathrm{T}$ stage and tumor response might be helpful to determine the adjuvant chemotherapy regimen.

\section{Conclusion}

An intensified regimen of concomitant boost radiotherapy plus concurrent capecitabine and oxaliplatin, followed by one cycle of Xelox, can be safely administered in patients with locally advanced rectal cancer, and produces a high rate of pCR. A prognostic score model is helpful to distinguish different long-term prognosis groups in early stage.

\section{Competing interests}

The authors declare that they have no competing interests.

\section{Authors' contributions}

ZJ and LFQ conceived and drafted the manuscript, ZZ and CSJ drafted and revised the manuscript, and all authors read and approved the final manuscript.

\section{Acknowledgement}

We thank Medjaden Bioscience Limited for assisting in the preparation of this manuscript.

\section{Author details}

${ }^{1}$ Department of Radiation Oncology, Fudan University Shanghai Cancer Center, No. 270, Dong'An Road, Shanghai 200032, China. ²Department of Colorectal Surgery, Fudan University Shanghai Cancer Center, No. 270, Dong'An Road, Shanghai 200032, China. ${ }^{3}$ Department of Pathology, Fudan 
University Shanghai Cancer Center, Shanghai 200032, China. ${ }^{4}$ Department of Nuclear Medicine, Fudan University Shanghai Cancer Center, Shanghai 200032, China. ${ }^{5}$ Department of Oncology, Shanghai Medical College, Fudan University, Shanghai 200032, China.

Received: 8 January 2014 Accepted: 21 February 2014 Published: 7 March 2014

\section{References}

1. Sauer R, Becker H, Hohenberger W, Rodel C, Wittekind C, Fietkau R, Martus P, Tschmelitsch J, Hager E, Hess CF, Karstens JH, Liersch T, Schmidberger H, Raab $R$ : Preoperative versus postoperative chemoradiotherapy for rectal cancer. N Engl J Med 2004, 351(17):1731-1740.

2. Quirke P, Durdey P, Dixon MF, Williams NS: Local recurrence of rectal adenocarcinoma due to inadequate surgical resection. Histopathological study of lateral tumour spread and surgical excision. Lancet 1986, 2(8514):996-999.

3. Bosset JF, Collette L, Calais G, Mineur L, Maingon P, Radosevic-Jelic L, Daban A, Bardet E, Beny A, Ollier JC: Chemotherapy with preoperative radiotherapy in rectal cancer. N Engl J Med 2006, 355(11):1114-1123.

4. Gerard JP, Conroy T, Bonnetain F, Bouche O, Chapet O, Closon-Dejardin MT, Untereiner M, Leduc B, Francois E, Maurel J, Seitz JF, Buecher B, Mackiewicz R, Ducreux $M$, Bedenne L: Preoperative radiotherapy with or without concurrent fluorouracil and leucovorin in T3-4 rectal cancers: results of FFCD 9203. J Clin Oncol 2006, 24(28):4620-4625.

5. Hartley A, Ho KF, McConkey C, Geh Jl: Pathological complete response following pre-operative chemoradiotherapy inrectal cancer: analysis of phase II/III trials. Br J Radiol 2005, 78(934):934-938.

6. Gerard JP, Azria D, Gourgou-Bourgade S, Martel-Laffay I, Hennequin C, Etienne PL, Vendrely V, Francois E, de La Roche G, Bouche O, Mirabel X, Denis B, Mineur L, Berdah JF, Mahe MA, Becouarn Y, Dupuis O, Lledo G, Montoto-Grillot $C$, Conroy T: Comparison of two neoadjuvant chemoradiotherapy regimens for locally advanced rectal cancer: results of the phase III trial ACCORD 12/0405-prodige 2. J Clin Oncol 2010, 28(10):1638-1644.

7. Aschele C, Cionini L, Lonardi S, Pinto C, Cordio S, Rosati G, Artale S, Tagliagambe A, Ambrosini G, Rosetti P, Bonetti A, Negru ME, Tronconi MC, Luppi G, Silvano G, Corsi DC, Bochicchio AM, Chiaulon G, Gallo M, Boni L: Primary tumor response to preoperative chemoradiation with or without oxaliplatinin locally advanced rectal cancer: pathologic results of the STAR-01 randomizedphase III trial. J Clin Oncol 2011, 29(20):2773-2780.

8. Roh MS, Gay OMJ: The impact of capecitabine and oxaliplatin in the preoperative multimodality treatment in patients with carcinoma of the rectum: NSABP R-04. J Clin Oncol 2011, 29(suppl):abstr 3503.

9. Rodel C, Liersch T, Becker H, Fietkau R, Hohenberger W, Hothorn T, Graeven U, Arnold D, Lang-Welzenbach M, Raab HR, Sulberg H, Wittekind C, Potapov S, Staib L, Hess C, Weigang-Kohler K, Grabenbauer GG, Hoffmanns H, Lindemann F, Schlenska-Lange A, Folprecht G, Sauer R: Preoperative chemoradiotherapy and postoperative chemotherapy with fluorouraciland oxaliplatin versus fluorouracil alone in locally advanced rectal cancer: initial results of the German CAO/ARO/AIO-04 randomised phase 3 trial. Lancet Oncol 2012, 13(7):679-687.

10. H-J Schmoll KH, Price T: Preoperative chemoradiotherapy and postoperative chemotherapy with capecitabine and oxaliplatin versus capecitabine alone in locally advanced rectal cancer: first results of the PETACC- 6 randomized phase III trial. J Clin Oncol 2013, 31(suppl):abstr 3531.

11. Yothers G, O'Connell MJ, Allegra CJ, Kuebler JP, Colangelo LH, Petrelli NJ, Wolmark N: Oxaliplatin as adjuvant therapy for colon cancer: updated results of NSABP C-07 trial, including survival and subset analyses. J Clin Oncol 2011, 29(28):3768-3774.

12. Sargent $D$, Shi $Q$, Yothers $G$, Van Cutsem E, Cassidy J, Saltz L, Wolmark N, Bot B, Grothey A, Buyse M, de Gramont A: Two or three year disease-free survival (DFS) as a primary end-point in stage III adjuvant colon cancer trials with fluoropyrimidines with or without oxaliplatin or irinotecan: data from 12,676 patients from MOSAIC, X-ACT, PETACC-3, C-06, C-07 and C89803. Eur J Cancer 2011, 47(7):990-996.

13. Gunderson LL, Sargent DJ, Tepper JE, Wolmark N, O'Connell MJ, Begovic M, Allmer C, Colangelo L, Smalley SR, Haller DG, Martenson JA, Mayer RJ, Rich TA, Ajani JA, MacDonald JS, Willett CG, Goldberg RM: Impact of T and N stage and treatment on survival and relapse in adjuvant rectal cancer: a pooled analysis. J Clin Oncol 2004, 22(10):1785-1796.
14. Guerrero Urbano MT, Henrys AJ, Adams EJ, Norman AR, Bedford $J$, Harrington KJ, Nutting CM, Dearnaley DP, Tait DM: Intensity-modulated radiotherapy in patients with locally advanced rectal cancer reduces volume of bowel treated to high dose levels. Int J Radiat Oncol Biol Phys 2006, 65(3):907-916.

15. Arbea L, Ramos LI, Martinez-Monge R, Moreno M, Aristu J: Intensity-modulated radiation therapy (IMRT) vs. 3D conformal radiotherapy (3DCRT) in locally advanced rectal cancer (LARC): dosimetric comparison and clinical implications. Radiat Oncol 2010, 5:17.

16. Caravatta L, Padula GD, Picardi V, Macchia G, Deodato F, Massaccesi M, Sofo L, Pacelli F, Rotondi F, Cecere G, Sallustio G, Di Lullo L, Piscopo A, Mignogna S, Bonomo P, Cellini N, Valentini V, Morganti AG: Concomitant boost radiotherapy and multidrug chemotherapy in the neoadjuvant treatment of locally advanced rectal cancer: results of a phase II study. Acta Oncol 2011, 50(8):1151-1157.

17. Mok H, Crane CH, Palmer MB, Briere TM, Beddar S, Delclos ME, Krishnan S, Das P: Intensity modulated radiation therapy (IMRT): differences in target volumes andimprovement in clinically relevant doses to small bowel in rectal carcinoma. Radiat Oncol 2011, 6:63.

18. Wolff HA, Wagner DM, Conradi LC, Hennies S, Ghadimi M, Hess CF, Christiansen $\mathrm{H}$ : Irradiation with protons for the individualized treatment of patients with locally advanced rectal cancer: a planning study with clinical implications. Radiother Oncol 2012, 102(1):30-37.

19. Hodapp N: [The ICRU Report 83: prescribing, recording and reporting photon-beam intensity-modulated radiation therapy (IMRT)]. Strahlenther Onkol 2012, 188(1):97-99.

20. Roels S, Duthoy W, Haustermans K, Penninckx F, Vandecaveye V, Boterberg T, De Neve W: Definition and delineation of the clinical target volume for rectal cancer. Int J Radiat Oncol Biol Phys 2006, 65(4):1129-1142.

21. Dworak O, Keilholz L, Hoffmann A: Pathological features of rectal cancer after preoperative radiochemotherapy. Int J Colorectal Dis 1997, 12(1):19-23.

22. Zhu J, Gu W, Lian P, Sheng W, Cai G, Shi D, Cai S, Zhang Z: A phase II trial of neoadjuvant IMRT-based chemoradiotherapy followed by onecycle of capecitabine for stage II/III rectal adenocarcinoma. Radiat Oncol 2013, 8(1):130.

23. Zhu J, Lian P, Liu F, Xu Y, Xu J, Guan Z, Liang L, Wang M, Cai S, Zhang Z: Phase II trial of first-line chemoradiotherapy with intensity-modulated radiationtherapy followed by chemotherapy for synchronous unresectable distant metastases rectal adenocarcinoma. Radiat Oncol 2013, 8:10.

24. Collette L, Bosset JF, den Dulk M, Nguyen F, Mineur L, Maingon P, Radosevic-Jelic $L$, Pierart M, Calais G: Patients with curative resection of cT3-4 rectal cancer after preoperative radiotherapy or radiochemotherapy: does anybody benefit from adjuvant fluorouracil-based chemotherapy? A trial of the European Organisation for Research and Treatment of Cancer Radiation Oncology Group. J Clin Oncol 2007, 25(28):4379-4386.

25. Capirci C, Valentini V, Cionini L, De Paoli A, Rodel C, Glynne-Jones R, Coco C, Romano M, Mantello G, Palazzi S, Mattia FO, Friso ML, Genovesi D, Vidali C, Gambacorta MA, Buffoli A, Lupattelli M, Favretto MS, La Torre G: Prognostic value of pathologic complete response after neoadjuvant therapy in locally advanced rectal cancer: long-term analysis of 566 ypCR patients. Int J Radiat Oncol Biol Phys 2008, 72(1):99-107.

26. Park IJ, You YN, Agarwal A, Skibber JM, Rodriguez-Bigas MA, Eng C, Feig BW, Das P, Krishnan S, Crane CH, Hu CY, Chang GJ: Neoadjuvant treatment response as an early response indicator for patients with rectal cancer. J Clin Oncol 2012, 30(15):1770-1776.

\section{doi:10.1186/1748-717X-9-70}

Cite this article as: Zhu et al.: Concomitant boost IMRT-based neoadjuvant chemoradiotherapy for clinical stage II/III rectal adenocarcinoma: results of a phase II study. Radiation Oncology 2014 9:70. 